\title{
Must Quantum Spacetimes Be Euclidean?
}

\author{
N. Pinto-Neto* \\ and \\ E. Sergio Santini ${ }^{\dagger}$ \\ Centro Brasileiro de Pesquisas Físicas \\ Rua Xavier Sigaud, 150 - Urca \\ 22290-180, Rio de Janeiro, RJ \\ Brazil
}

June 19, 2018

*e-mail adress: nelsonpn@lafex.cbpf.br

${ }^{\dagger}$ e-mail adress: santini@lafex.cbpf.br 


\begin{abstract}
The Bohm-de Broglie interpretation of quantum mechanics is applied to canonical quantum cosmology. It is shown that, irrespective of any regularization or choice of factor ordering of the Wheeler-DeWitt equation, the unique relevant quantum effect which does not break spacetime is the change of its signature from lorentzian to euclidean. The other quantum effects are either trivial or break the four-geometry of spacetime. A Bohm-de Broglie picture of a quantum geometrodynamics is constructed, which allows the investigation of these latter structures. For instance, it is shown that any real solution of the Wheeler-De Witt equation yields a generate four-geometry compatible with the strong gravity limit of General Relativity and the Carroll group. Due to the more detailed description of quantum geometrodynamics given by the Bohm-de Broglie interpretation, some new boundary conditions on solutions of the Wheeler-DeWitt equation must be imposed in order to preserve consistency of this finer view.
\end{abstract}

PACS number(s): 98.80.Hw, 04.60.Kz, 04.20.Cv. 


\section{Introduction}

Almost all physicists believe that quantum mechanics is a universal and fundamental theory, applicable to any physical system, from which classical physics can be recovered. The Universe is, of course, a valid physical system: there is a theory, Standard Cosmology, which is able to describe it in physical terms, and make predictions which can be confirmed or refuted by observations. In fact, the observations until now confirm the standard cosmological scenario. Hence, supposing the universality of quantum mechanics, the Universe itself must be described by quantum theory, from which we could recover Standard Cosmology. However, the Copenhaguen interpretation of quantum mechanics [1, 2, 3], which is the one taught in undergraduate courses and employed by the majority of physicists in all areas (specially the von Neumann's approach), cannot be used in a Quantum Theory of Cosmology. This is because it imposes the existence of a classical domain. In von Neumann's view, for instance, the necessity of a classical domain comes from the way it solves the measurement problem (see Ref. [4] for a good discussion). In an impulsive measurement of some observable, the wave function of the observed system plus macroscopic apparatus splits into many branches which almost do not overlap (in order to be a good measurement), each one containing the observed system in an eigenstate of the measured observable,

\footnotetext{
${ }^{1}$ Athough these three authors have different views from quantum theory, the first emphasizing the indivisibility of quantum phenomena, the second with his notion of potentiality, and the third with the concept of quantum states, for all of them the existence of a classical domain is crucial. That is why we group their approaches under the same name "Copenhaguen interpretation".
} 
and the pointer of the apparatus pointing to the respective eigenvalue. However, in the end of the measurement, we observe only one of these eigenvalues, and the measurement is robust in the sense that if we repeat it immediately after, we obtain the same result. So it seems that the wave function collapses, the other branches disappear. The Copenhaguen interpretation assumes that this collapse is real. However, a real collapse cannot be described by the unitary Schrödinger evolution. Hence, the Copenhaguen interpretation must assume that there is a fundamental process in a measurement which must occur outside the quantum world, in a classical domain. Of course, if we want to quantize the whole Universe, there is no place for a classical domain outside it, and the Copenhaguen interpretation cannot be applied. Note that decoherence cannot solve this problem [5, 6]. It can explain why the splitting of the wave function is given in terms of the pointer basis states, and why we do not see superpositions of macroscopic objects, due to the effective diagonalization of the reduced density matrix. However it does not explain the collapse after the measurement is completed, or why all but one of the diagonal elements of the density matrix become null when the measurement is finished. Hence, if someone insists with the Copenhaguen interpretation, she or he must assume that quantum theory is not universal, or at least try to improve it by means of further concepts like in the consistent histories approach [7], which is however incomplete until now. Nevertheless, there are some ways out from this dilemma. We can say that the Schrödinger evolution is an approximation of a more fundamental non-linear theory which can accomplish the collapse 8, 9], or that the collapse is effective but not real, in the sense that the other branches disappear from the observer but do 
not disappear from existence. In this second category we can cite the ManyWorlds Interpretation [10] and the Bohm-de Broglie Interpretation [11, 12]. In the former, all the possibilities in the splitting are actually realized. In each branch there is an observer with the knowledge of the corresponding eigenvalue of this branch, but she or he is not aware of the other observers and the other possibilities because the branches do not interfere. In the latter, a point-particle in configuration space describing the observed system and apparatus is supposed to exist, independently on any observations. In the splitting, this point particle will enter into one of the branches (which one depends on the initial position of the point particle before the measurement, which is unknown), and the other branches will be empty. It can be shown [12] that the empty waves can neither interact with other particles, nor with the point particle containing the apparatus. Hence, no observer can be aware of the other branches which are empty. Again we have an effective but not real collapse (the empty waves continue to exist), but now with no multiplication of observers. Of course these interpretations can be used in quantum cosmology. Schrödinger evolution is always valid, and there is no need of a classical domain outside the observed system.

In this paper we will foccus on the application of the Bohm-de Broglie interpretation to quantum cosmology [13, 14, 15, 16]. In this approach, the fundamental object of quantum gravity, the geometry of 3-dimensional spacelike hypersurfaces, is supposed to exist independently on any observation or measurement, as well as its canonical momentum, the extrinsic curvature of the spacelike hypersurfaces. Its evolution, labeled by some time parameter, is dictated by a quantum evolution that is different from the classical 
one due to the presence of a quantum potential which appears naturally from the Wheeler-DeWitt equation. This interpretation has been applied to many minisuperspace models $[13,16,17,18,19,20]$, obtained by the imposition of homogeneity of the spacelike hypersurfaces. The classical limit, the singularity problem, the cosmological constant problem, and the time issue have been discussed. For instance, in some of these papers it was shown that in models involving scalar fields or radiation, which are nice representatives of the matter content of the early universe, the singularity can be clearly avoided by quantum effects. In the Bohm-de Broglie interpretation description, the quantum potential becomes important near the singularity, yielding a repulsive quantum force counteracting the gravitational field, avoiding the singularity and yielding inflation. The classical limit (given by the limit where the quantum potential becomes negligible with respect to the classical energy) for large scale factors are usually attainable, but for some scalar field models it depends on the quantum state and initial conditions. In fact it is possible to have small classical universes and large quantum ones [20]. About the time issue, it was shown that for any choice of the lapse function the quantum evolution of the homogeneous hypersurfaces yield the same four-geometry [16]. What remained to be studied is if this fact remains valid in the full theory, where we are not restricted to homogeneous spacelike hypersurfaces. The question is, given an initial hypersurface with consistent initial conditions, does the evolution of the initial three-geometry driven by the quantum bohmian dynamics yields the same four-geometry for any choice of the lapse and shift functions? We know that this is true if the three-geometry is evolved by the dynamics of classical General Relativity 
(GR), but it can be false if the evolving dynamics is the quantum bohmian one. The purpose of this paper is to study and answer this question in detail. The idea is to put the quantum bohmian dynamics in hamiltonian form, and then use strong results presented in the literature exhibiting the most general form that a hamiltonian should have in order to form a fourgeometry from the evolution of 3-geometries [21]. Our conclusion is that, in general, the quantum bohmian evolution of the 3-geometries does not yield any four-geometry at all. Only for very special quantum states a quantum four-geometry can be obtained, and it must be euclidean. Hence, our answer to the question in the title is in the affirmative. More important, we arrive at these conclusions without assuming any regularization and factor ordering of the Wheeler-DeWitt equation. As we know, the Wheeler-DeWitt equation involves the application of the product of local operators on states at the same space point, which is ill defined [22]. Hence we need to regularize it in order to solve the factor ordering problem, and have a theory free of anomalies (for some proposals, see Refs [23, 24, 25]). Our conclusions are completly independent on these issues. Also, even in the general case where there is no four-geometry, we can obtain a picture of the quantum structure yielded by the bohmian dynamics, which is not a spacetime but something else, as the generate 4-geometries compatible with the Carroll group [26].

This paper is organized as follows: in the next section we review the Bohm-de Broglie interpretation of quantum mechanics for non-relativistic particles and quantum field theory in flat spacetime. In section 3 we apply the Bohm-de Broglie interpretation to canonical quantum gravity. In section 4 we prove that the bohmian evolution of the 3-geometries, irrespective of 
any regularization and factor ordering of the Wheeler-DeWitt equation, can be obtained from a specific hamiltonian, which is of course different from the classical one. We then use this hamiltonian to obtain the main results of our paper concerning the possibilities of obtaining quantum 4-geometries, and the procedure of obtaining a picture of other quantum structures. The classical limits of these many possibilities are also discussed. We end with conclusions and many perspectives for future work. In the appendix we present a concrete midisuperspace example of some quantum states which illustrates the discussion of section 4 .

\section{The Bohm-de Broglie Interpretation}

In this section we will review the Bohm-de Broglie interpretation of quantum mechanics. We will first show how this interpretation works in the case of a single particle described by a Schrödinger equation, and then we will obtain, by analogy, the causal interpretation of quantum field theory in flat spacetime.

Let us begin with the Bohm-de Broglie interpretation of the Schrödinger equation describing a single particle. In the coordinate representation, for a non-relativistic particle with Hamiltonian $H=p^{2} / 2 m+V(x)$, the Schrödinger equation is

$$
i \hbar \frac{\partial \Psi(x, t)}{\partial t}=\left[-\frac{\hbar^{2}}{2 m} \nabla^{2}+V(x)\right] \Psi(x, t) .
$$

We can transform this differential equation over a complex field into a pair 
of coupled differential equations over real fields, by writing $\Psi=A \exp (i S / \hbar)$, where $A$ and $S$ are real functions, and substituting it into (1). We obtain the following equations.

$$
\begin{gathered}
\frac{\partial S}{\partial t}+\frac{(\nabla S)^{2}}{2 m}+V-\frac{\hbar^{2}}{2 m} \frac{\nabla^{2} A}{A}=0, \\
\frac{\partial A^{2}}{\partial t}+\nabla \cdot\left(A^{2} \frac{\nabla S}{m}\right)=0 .
\end{gathered}
$$

The usual probabilistic interpretation, i.e. the Copenhagen interpretation, understands equation (3) as a continuity equation for the probability density $A^{2}$ for finding the particle at position $x$ and time $t$. All physical information about the system is contained in $A^{2}$, and the total phase $S$ of the wave function is completely irrelevant. In this interpretation, nothing is said about $S$ and its evolution equation (2). However, when the term $\frac{\hbar^{2}}{2 m} \frac{\nabla^{2} A}{A}$ is negligible, we can interpret Eqs. (2) and (3) as equations for an ensemble of classical particles under the influence of a classical potential $V$ through the HamiltonJacobi equation (2), whose probability density distribution in space $A^{2}(x, t)$ satisfies the continuity equation (3), where $\nabla S(x, t) / m$ is the velocity field $v(x, t)$ of the ensemble of particles. When $\frac{\hbar^{2}}{2 m} \frac{\nabla^{2} A}{A}$ is not negligible, we can still understand Eq. (2) as a Hamilton-Jacobi equation for an ensemble of particles. However, their trajectories are no more the classical ones, due to the presence of the quantum potential term in Eq. (2).

The Bohm-de Broglie interpretation of quantum mechanics is based on the two equations (2) and (3) in the way outlined above, not only on the last one as it is the Copenhagen interpretation. The starting idea is that the position $x$ and momentum $p$ are always well defined, with the particle's 
path being guided by a new field, the quantum field. The field $\Psi$ obeys Schrödinger equation (11), which can be written as the two real equations (2) and (3). Equation (2) is interpreted as a Hamilton-Jacobi type equation for the quantum particle subjected to an external potential, which is the classical potential plus the new quantum potential

$$
Q \equiv-\frac{\hbar^{2}}{2 m} \frac{\nabla^{2} A}{A}
$$

Once the field $\Psi$, whose effect on the particle trajectory is through the quantum potential (雨), is obtained from Schrödinger equation, we can also obtain the particle trajectory, $x(t)$, by integrating the differential equation $p=m \dot{x}=\nabla S(x, t)$, which is called the guidance relation (a dot means time derivative). Of course, from this differential equation, the non-classical trajectory $x(t)$ can only be known if the initial position of the particle is given. However, we do not know the initial position of the particle because we do not know how to measure it without disturbances (it is the hidden variable of the theory). To agree with quantum mechanical experiments, we have to postulate that, for a statistical ensemble of particles in the same quantum field $\Psi$, the probability density distribution of initial positions $x_{0}$ is $P\left(x_{0}, t_{0}\right)=A^{2}\left(x_{0}, t=t_{0}\right)$. Equation (3) guarantees that $P(x, t)=A^{2}(x, t)$ for all times. In this way, the statistical predictions of quantum theory in the Bohm-de Broglie interpretation are the same as in the Copenhaguen interpretation?

\footnotetext{
${ }^{2}$ It has been shown that under typical chaotic situations, and only within the Bohm-de Broglie interpretation, a probability distribution $P \neq A^{2}$ would rapidly approach the value $P=A^{2}[27,28]$. In this case, the probability postulate would be unnecessary, and we
} 
It is interesting to note that the quantum potential depends only on the form of $\Psi$, not on its absolute value, as can be seen from equation (4). This fact brings home the non-local and contextual character of the quantum potential' This is a necessary feature because Bell's inequalities together with Aspect's experiments show that, in general, a quantum theory must be either non-local or non-ontological. As the Bohm-de Broglie interpretation is ontological, than it must be non-local, as it is. The non-local and contextual quantum potential causes the quantum effects.

The function $A$ plays a dual role in the Bohm-de Broglie interpretation: it gives the quantum potential and the probability density distribution of positions, but this last role is secondary. If in some model there is no notion of probability, we can still get information from the system using the guidance relations. In this case, $A^{2}$ does not need to be normalizable. The Bohm-de Broglie interpretation is not, in essence, a probabilistic interpretation. It is straightforward to apply it to a single system.

The classical limit can be obtained in a very simple way. We only have to find the conditions for having $Q=0$. There is no need to have a classical domain because this interpretation is ontological. The question on why in a real measurement we see an effective collapse of the wave function is answered by noting that, in a measurement, the wave function splits in a superposition of non-overlapping branches. Hence the point particle (representing the could have situations, in very short time intervals, where this modified Bohm-de Broglie interpretation would differ from the Copenhaguen interpretation.

${ }^{3}$ The non-locality of $Q$ becomes evident when we generalize the causal interpretation to a many particles system. 
particle being measured plus the macroscopic apparatus) will enter into one particular branch, which one depends on the initial conditions, and it will be influenced by the quantum potential related only with this branch, which is the only one that is not negligible in the region where the point particle actually is. The other empty branches continue to exist, but they neither influence on the point particle nor on any other particle [12]. There is an effective but not real collapse. The Schrödinger equation is always valid.

For quantum fields in flat spacetime, we can apply a similar reasoning. As an example, take the Schrödinger functional equation for a quantum scalar field:

$$
i \hbar \frac{\partial \Psi(\phi, t)}{\partial t}=\int d^{3} x\left\{\frac{1}{2}\left[-\hbar^{2} \frac{\delta^{2}}{\delta \phi^{2}}+(\nabla \phi)^{2}\right]+U(\phi)\right\} \Psi(\phi, t) .
$$

Writing again the wave functional as $\Psi=A \exp (i S / \hbar)$, we obtain:

$$
\begin{gathered}
\frac{\partial S}{\partial t}+\int d^{3} x\left\{\frac{1}{2}\left[\left(\frac{\delta S}{\delta \phi}\right)^{2}+(\nabla \phi)^{2}\right]+U(\phi)+Q(\phi)\right\}=0, \\
\frac{\partial A^{2}}{\partial t}+\int d^{3} x \frac{\delta}{\delta \phi}\left(A^{2} \frac{\delta S}{\delta \phi}\right)=0,
\end{gathered}
$$

where $Q(\phi)=-\hbar^{2} \frac{1}{2 A} \frac{\delta^{2} A}{\delta \phi^{2}}$ is the corresponding (unregulated) quantum potential. The first equation is viewed as a modified Hamilton-Jacobi equation governing the evolution of some initial field configuration through time, which will be different from the classical one due to the presence of the quantum potential. The guidance relation is now given by

$$
\Pi_{\phi}=\dot{\phi}=\frac{\delta S}{\delta \phi}
$$

The second equation is the continuity equation for the probability density $A^{2}\left[\phi(x), t_{0}\right]$ of having the initial field configuration at time $t_{0}$ given by $\phi(x)$. 
A detailed analysis of the Bohm-de Broglie interpretation of quantum field theory is given in Ref. [29] for the case of quantum electrodynamics.

\section{The Bohm-de Broglie Interpretation of Canon- ical Quantum Cosmology}

Let us now apply the Bohm-de Broglie interpretation to canonical quantum cosmology. We will quantize General Relativity Theory (GR) where the matter content is a minimally coupled scalar field with arbitrary potential. All subsequent results remain essentially the same for any matter field which couples uniquely with the metric, not with their derivatives.

The classical hamiltonian of GR with a scalar field is given by:

$$
H=\int d^{3} x\left(N \mathcal{H}+N^{j} \mathcal{H}_{j}\right)
$$

where

$$
\begin{aligned}
\mathcal{H}= & \kappa G_{i j k l} \Pi^{i j} \Pi^{k l}+\frac{1}{2} h^{-1 / 2} \Pi_{\phi}^{2}+ \\
& +h^{1 / 2}\left[-\kappa^{-1}\left(R^{(3)}-2 \Lambda\right)+\frac{1}{2} h^{i j} \partial_{i} \phi \partial_{j} \phi+U(\phi)\right] \\
\mathcal{H}_{j}= & -2 D_{i} \Pi_{j}^{i}+\Pi_{\phi} \partial_{j} \phi .
\end{aligned}
$$

In these equations, $h_{i j}$ is the metric of closed 3-dimensional spacelike hypersurfaces, and $\Pi^{i j}$ is its canonical momentum given by

$$
\Pi^{i j}=-h^{1 / 2}\left(K^{i j}-h^{i j} K\right)=G^{i j k l}\left(\dot{h}_{k l}-D_{k} N_{l}-D_{l} N_{k}\right),
$$

where

$$
K_{i j}=-\frac{1}{2 N}\left(\dot{h}_{i j}-D_{i} N_{j}-D_{j} N_{i}\right)
$$


is the extrinsic curvature of the hypersurfaces (indices are raisen and lowered by the 3 -metric $h_{i j}$ and its inverse $h^{i j}$ ). The canonical momentum of the scalar field is now

$$
\Pi_{\phi}=\frac{h^{1 / 2}}{N}\left(\dot{\phi}-N^{i} \partial_{i} \phi\right) .
$$

The quantity $R^{(3)}$ is the intrinsic curvature of the hypersurfaces and $h$ is the determinant of $h_{i j}$. The lapse function $N$ and the shift function $N_{j}$ are the Lagrange multipliers of the super-hamiltonian constraint $\mathcal{H} \approx 0$ and the super-momentum constraint $\mathcal{H}^{j} \approx 0$, respectively. They are present due to the invariance of GR under spacetime coordinate transformations. The quantities $G_{i j k l}$ and its inverse $G^{i j k l}\left(G_{i j k l} G^{i j a b}=\delta_{k l}^{a b}\right)$ are given by

$$
\begin{aligned}
G^{i j k l} & =\frac{1}{2} h^{1 / 2}\left(h^{i k} h^{j l}+h^{i l} h^{j k}-2 h^{i j} h^{k l}\right), \\
G_{i j k l} & =\frac{1}{2} h^{-1 / 2}\left(h_{i k} h_{j l}+h_{i l} h_{j k}-h_{i j} h_{k l}\right),
\end{aligned}
$$

which is called the DeWitt metric. The quantity $D_{i}$ is the $i$-component of the covariant derivative operator on the hypersurface, and $\kappa=16 \pi G / c^{4}$.

The classical 4-metric

$$
d s^{2}=-\left(N^{2}-N^{i} N_{i}\right) d t^{2}+2 N_{i} d x^{i} d t+h_{i j} d x^{i} d x^{j}
$$

and the scalar field which are solutions of the Einstein's equations can be obtained from the Hamilton's equations of motion

$$
\begin{aligned}
\dot{h}_{i j} & =\left\{h_{i j}, H\right\}, \\
\dot{\Pi}^{i j} & =\left\{\Pi^{i j}, H\right\}, \\
\dot{\phi} & =\{\phi, H\},
\end{aligned}
$$




$$
\dot{\Pi}_{\phi}=\left\{\Pi_{\phi}, H\right\}
$$

for some choice of $N$ and $N^{i}$, and if we impose initial conditions compatible with the constraints

$$
\begin{aligned}
\mathcal{H} & \approx 0, \\
\mathcal{H}_{i} & \approx 0 .
\end{aligned}
$$

It is a feature of the hamiltonian of GR that the 4-metrics (17) constructed in this way, with the same initial conditions, describe the same four-geometry for any choice of $N$ and $N^{i}$.

The algebra of the constraints close in the following form (we follow the notation of Ref. [21]):

$$
\begin{aligned}
\left\{\mathcal{H}(x), \mathcal{H}\left(x^{\prime}\right)\right\} & =\mathcal{H}^{i}(x) \partial_{i} \delta^{3}\left(x, x^{\prime}\right)-\mathcal{H}^{i}\left(x^{\prime}\right) \partial_{i} \delta^{3}\left(x^{\prime}, x\right) \\
\left\{\mathcal{H}_{i}(x), \mathcal{H}\left(x^{\prime}\right)\right\} & =\mathcal{H}(x) \partial_{i} \delta^{3}\left(x, x^{\prime}\right) \\
\left\{\mathcal{H}_{i}(x), \mathcal{H}_{j}\left(x^{\prime}\right)\right\} & =\mathcal{H}_{i}(x) \partial_{j} \delta^{3}\left(x, x^{\prime}\right)+\mathcal{H}_{j}\left(x^{\prime}\right) \partial_{i} \delta^{3}\left(x, x^{\prime}\right)
\end{aligned}
$$

To quantize this constrained system, we follow the Dirac quantization procedure. The constraints become conditions imposed on the possible states of the quantum system, yielding the following quantum equations:

$$
\begin{aligned}
& \hat{\mathcal{H}}_{i} \mid \Psi>=0 \\
& \hat{\mathcal{H}} \mid \Psi>=0
\end{aligned}
$$

In the metric and field representation, the first equation is

$$
-2 h_{l i} D_{j} \frac{\delta \Psi\left(h_{i j}, \phi\right)}{\delta h_{l j}}+\frac{\delta \Psi\left(h_{i j}, \phi\right)}{\delta \phi} \partial_{i} \phi=0,
$$


which implies that the wave functional $\Psi$ is an invariant under space coordinate transformations.

The second equation is the Wheeler-DeWitt equation [30, 31]. Writing it unregulated in the coordinate representation we get

$$
\left\{-\hbar^{2}\left[\kappa G_{i j k l} \frac{\delta}{\delta h_{i j}} \frac{\delta}{\delta h_{k l}}+\frac{1}{2} h^{-1 / 2} \frac{\delta^{2}}{\delta \phi^{2}}\right]+V\right\} \Psi\left(h_{i j}, \phi\right)=0,
$$

where $V$ is the classical potential given by

$$
V=h^{1 / 2}\left[-\kappa^{-1}\left(R^{(3)}-2 \Lambda\right)+\frac{1}{2} h^{i j} \partial_{i} \phi \partial_{j} \phi+U(\phi)\right] .
$$

This equation involves products of local operators at the same space point, hence it must be regularized. After doing this, one should find a factor ordering which makes the theory free of anomalies, in the sense that the commutator of the operator version of the constraints close in the same way as their respective classical Poisson brackets (24). Hence, Eq. (28) is only a formal one which must be worked out [23, 24, 25].

Let us now see what is the Bohm-de Broglie interpretation of the solutions of Eqs. (25) and (26) in the metric and field representation. First we write the wave functional in polar form $\Psi=A \exp (i S / \hbar)$, where $A$ and $S$ are functionals of $h_{i j}$ and $\phi$. Substituting it in Eq. (27), we get two equations saying that $A$ and $S$ are invariant under general space coordinate transformations:

$$
\begin{aligned}
& -2 h_{l i} D_{j} \frac{\delta S\left(h_{i j}, \phi\right)}{\delta h_{l j}}+\frac{\delta S\left(h_{i j}, \phi\right)}{\delta \phi} \partial_{i} \phi=0, \\
& -2 h_{l i} D_{j} \frac{\delta A\left(h_{i j}, \phi\right)}{\delta h_{l j}}+\frac{\delta A\left(h_{i j}, \phi\right)}{\delta \phi} \partial_{i} \phi=0 .
\end{aligned}
$$

The two equations we obtain for $A$ and $S$ when we substitute $\Psi=$ $A \exp (i S / \hbar)$ into Eq. (26) will of course depend on the factor ordering we 
choose. However, in any case, one of the equations will have the form

$$
\kappa G_{i j k l} \frac{\delta S}{\delta h_{i j}} \frac{\delta S}{\delta h_{k l}}+\frac{1}{2} h^{-1 / 2}\left(\frac{\delta S}{\delta \phi}\right)^{2}+V+Q=0
$$

where $V$ is the classical potential given in Eq. (29). Contrary to the other terms in Eq. (32), which are already well defined, the precise form of $Q$ depends on the regularization and factor ordering which are prescribed for the Wheeler-DeWitt equation. In the unregulated form given in Eq. (28), $Q$ is

$$
Q=-\hbar^{2} \frac{1}{A}\left(\kappa G_{i j k l} \frac{\delta^{2} A}{\delta h_{i j} \delta h_{k l}}+\frac{h^{-1 / 2}}{2} \frac{\delta^{2} A}{\delta \phi^{2}}\right) .
$$

Also, the other equation besides (32) in this case is

$$
\kappa G_{i j k l} \frac{\delta}{\delta h_{i j}}\left(A^{2} \frac{\delta S}{\delta h_{k l}}\right)+\frac{1}{2} h^{-1 / 2} \frac{\delta}{\delta \phi}\left(A^{2} \frac{\delta S}{\delta \phi}\right)=0 .
$$

Let us now implement the Bohm-de Broglie interpretation for canonical quantum gravity. First of all we note that Eqs. (30) and (32), which are always valid irrespective of any factor ordering of the Wheeler-DeWitt equation, are like the Hamilton-Jacobi equations for GR, suplemented by an extra term $Q$ in the case of Eq. (32), which we will call the quantum potential. By analogy with the cases of non-relativistic particle and quantum field theory in flat spacetime, we will postulate that the 3-metric of spacelike hypersurfaces, the scalar field, and their canonical momenta always exist, independent on any observation, and that the evolution of the 3-metric and scalar field can be obtained from the guidance relations

$$
\Pi^{i j}=\frac{\delta S\left(h_{a b}, \phi\right)}{\delta h_{i j}}
$$




$$
\Pi_{\phi}=\frac{\delta S\left(h_{i j}, \phi\right)}{\delta \phi},
$$

with $\Pi^{i j}$ and $\Pi_{\phi}$ given by Eqs. (12) and (14), respectively. Like before, these are first order differential equations which can be integrated to yield the 3-metric and scalar field for all values of the $t$ parameter. These solutions depend on the initial values of the 3-metric and scalar field at some initial hypersurface. The evolution of these fields will of course be different from the classical one due to the presence of the quantum potential term $Q$ in Eq. (32). The classical limit is once more conceptually very simple: it is given by the limit where the quantum potential $Q$ becomes negligible with respect to the classical energy. The only difference from the previous cases of the non-relativistic particle and quantum field theory in flat spacetime is the fact that the equivalent of Eqs. (3) and (7) for canonical quantum gravity, which in the naive ordering is Eq. (34), cannot be interpreted as a continuity equation for a probabiblity density $A^{2}$ because of the hyperbolic nature of the DeWitt metric $G_{i j k l}$. However, even without a notion of probability, which in this case would mean the probability density distribution for initial values of the 3-metric and scalar field in an initial hypersurface, we can extract a lot of information from Eq. (32) whatever is the quantum potential $Q$, as will see now. After we get these results, we will return to this probability issue in the last section.

First we note that, whatever is the form of the quantum potential $Q$, it must be a scalar density of weight one. This comes from the Hamilton-Jacobi equation (32). From this equation we can express $Q$ as

$$
Q=-\kappa G_{i j k l} \frac{\delta S}{\delta h_{i j}} \frac{\delta S}{\delta h_{k l}}-\frac{1}{2} h^{-1 / 2}\left(\frac{\delta S}{\delta \phi}\right)^{2}-V .
$$


As $S$ is an invariant (see Eq. (30), then $\delta S / \delta h_{i j}$ and $\delta S / \delta \phi$ must be a second rank tensor density and a scalar density, both of weight one, respectively. When their products are contracted with $G_{i j k l}$ and multiplied by $h^{-1 / 2}$, respectively, they form a scalar density of weight one. As $V$ is also a scalar density of weight one, then $Q$ must also be. Furthermore, $Q$ must depend only on $h_{i j}$ and $\phi$ because it comes from the wave functional which depends only on these variables. Of course it can be non-local (we show an example in the appendix), i.e., depending on integrals of the fields over the whole space, but it cannot depend on the momenta.

Now we will investigate the following important problem. From the guidance relations (35) and (36) we obtain the following first order partial differential equations:

$$
\dot{h}_{i j}=2 N G_{i j k l} \frac{\delta S}{\delta h_{k l}}+D_{i} N_{j}+D_{j} N_{i}
$$

and

$$
\dot{\phi}=N h^{-1 / 2} \frac{\delta S}{\delta \phi}+N^{i} \partial_{i} \phi
$$

The question is, given some initial 3-metric and scalar field, what kind of structure do we obtain when we integrate this equations in the parameter $t$ ? Does this structure form a 4-dimensional geometry with a scalar field for any choice of the lapse and shift functions? Note that if the functional $S$ were a solution of the classical Hamilton-Jacobi equation, which does not contain the quantum potential term, then the answer would be in the affirmative because we would be in the scope of GR. But $S$ is a solution of the modified Hamilton-Jacobi equation (32), and we cannot guarantee that this will continue to be true. We may obtain a complete different structure due 
to the quantum effects driven by the quantum potential term in Eq. (32). To answer this question we will move from this Hamilton-Jacobi picture of quantum geometrodynamics to a hamiltonian picture. This is because many strong results concerning geometrodynamics were obtained in this later picture [21, 32]. We will construct a hamiltonian formalism which is consistent with the guidance relations (35) and (36). It yields the bohmian trajectories (38) and (39) if the guidance relations are satisfied initially. Once we have this hamiltonian, we can use well known results in the literature to obtain strong results about the Bohm-de Broglie view of quantum geometrodynamics.

\section{The Bohm-de Broglie View of Quantum Geometrodynamics}

Examining Eqs. (30) and (32), we can easily guess that the hamiltonian which generates the bohmian trajectories, once the guidance relations (35) and (36) are satisfied initially, should be given by:

$$
H_{Q}=\int d^{3} x\left[N(\mathcal{H}+Q)+N^{i} \mathcal{H}_{i}\right]
$$

where we define

$$
\mathcal{H}_{Q} \equiv \mathcal{H}+Q
$$

The quantities $\mathcal{H}$ and $\mathcal{H}_{i}$ are the usual GR super-hamiltonian and supermomentum constraints given by Eqs. (10) and (11). In fact, the guidance relations (35) and (36) are consistent with the constraints $\mathcal{H}_{Q} \approx 0$ and $\mathcal{H}_{i} \approx 0$ 
because $S$ satisfies (30) and (32). Futhermore, they are conserved by the hamiltonian evolution given by (40). Let us see this in some detail.

First, we write Eqs. (35) and (36) in a sligthly different form defining

$$
\Phi^{i j} \equiv \Pi^{i j}-\frac{\delta S\left(h_{a b}, \phi\right)}{\delta h_{i j}} \approx 0
$$

and

$$
\Phi_{\phi} \equiv \Pi_{\phi}-\frac{\delta S\left(h_{i j}, \phi\right)}{\delta \phi} \approx 0 .
$$

Let us now calculate $\left\{\Phi^{i j}(x), \mathcal{H}_{Q}\left(x^{\prime}\right)\right\},\left\{\Phi_{\phi}(x), \mathcal{H}_{Q}\left(x^{\prime}\right)\right\},\left\{\Phi^{a b}(x), \mathcal{H}_{i}\left(x^{\prime}\right)\right\}$ and $\left\{\Phi_{\phi}(x), \mathcal{H}_{i}\left(x^{\prime}\right)\right\}$, and see if the guidance relations (42) and (43), now viewed as constraints, are conserved by the hamiltonian $H_{Q}$.

$$
\begin{aligned}
\left\{\mathcal{H}_{Q}(x), \Phi^{i j}\left(x^{\prime}\right)\right\}= & \kappa \frac{\delta G_{a b c d}}{\delta h_{i j}^{\prime}} \Pi^{a b} \Pi^{c d}+\frac{1}{2} \frac{\delta h^{-1 / 2}}{\delta h_{i j}^{\prime}} \Pi_{\phi}^{2}+\frac{\delta(V+Q)}{\delta h_{i j}^{\prime}} \\
& +2 \kappa G_{a b c d} \Pi^{a b} \frac{\delta^{2} S}{\delta h_{c d} \delta h_{i j}^{\prime}}+h^{-\frac{1}{2}} \Pi_{\phi} \frac{\delta^{2} S}{\delta \phi \delta h_{i j}^{\prime}} \\
= & \kappa \frac{\delta G_{a b c d}}{\delta h_{i j}^{\prime}}\left(\Phi^{a b} \Phi^{c d}+2 \Phi^{a b} \frac{\delta S}{\delta h_{c d}}\right)+\frac{1}{2} \frac{\delta h^{-\frac{1}{2}}}{\delta h_{i j}^{\prime}}\left(\Phi_{\phi}^{2}+2 \Phi_{\phi} \frac{\delta S}{\delta \phi}\right) \\
& +2 \kappa G_{a b c d} \Phi^{a b} \frac{\delta^{2} S}{\delta h_{c d} \delta h_{i j}^{\prime}}+h^{-\frac{1}{2}} \Phi_{\phi} \frac{\delta S}{\delta \phi \delta h_{i j}^{\prime}} \\
& +\frac{\delta}{\delta h_{i j}^{\prime}}\left[\kappa G_{a b c d} \frac{\delta S}{\delta h_{a b}} \frac{\delta S}{\delta h_{c d}}+\frac{1}{2} h^{-\frac{1}{2}}\left(\frac{\delta S}{\delta \phi}\right)^{2}+V+Q\right]
\end{aligned}
$$

where the primes denote evaluation at $x^{\prime}$.

The last term is zero because of Eq.(32), and we get

$$
\left\{\mathcal{H}_{Q}(x), \Phi^{i j}\left(x^{\prime}\right)\right\}=\left\{\kappa \left[-\frac{1}{2} G_{a b c d} h^{i j}\right.\right.
$$




$$
\begin{aligned}
& \left.+\frac{1}{2} h^{-\frac{1}{2}}\left(4 \delta_{a c}^{i j} h_{b d}-\delta_{a b}^{i j} h_{c d}-\delta_{c d}^{i j} h_{a b}\right)\right]\left(\Phi^{a b} \Phi^{c d}+2 \Phi^{a b} \frac{\delta S}{\delta h_{c d}}\right) \\
& \left.-\frac{1}{4} h^{-\frac{1}{2}} h^{i j}\left(\Phi_{\phi}^{2}+2 \Phi_{\phi} \frac{\delta S}{\delta \phi}\right)\right\} \delta^{3}\left(x, x^{\prime}\right) \\
& +2 \kappa G_{a b c d} \frac{\delta^{2} S}{\delta h_{c d} \delta h_{i j}^{\prime}} \Phi^{a b}+h^{-\frac{1}{2}} \frac{\delta^{2} S}{\delta \phi \delta h_{i j}^{\prime}} \Phi_{\phi} \approx 0 .
\end{aligned}
$$

In the same way we can prove that

$$
\left\{\mathcal{H}_{Q}(x), \Phi_{\phi}\left(x^{\prime}\right)\right\}=2 \kappa G_{a b c d} \frac{\delta^{2} S}{\delta h_{a b} \delta \phi^{\prime}} \Phi^{c d}+h^{-\frac{1}{2}} \frac{\delta^{2} S}{\delta \phi \delta \phi^{\prime}} \Phi_{\phi} \approx 0
$$

where we have used that the functional derivative of Eq. (32) with respect to $\phi$ is zero.

For the Poisson brackets involving the supermomentum constraint, as $S$ is an invariant because it satisfies Eq. (30), then $\Phi^{i j}$ and $\Phi_{\phi}$ are a second rank tensor density and a scalar density, respectively, both of weigth one. As $\mathcal{H}_{i}$ is the generator of space coordinate transformations, we get

$$
\left\{\mathcal{H}_{i}(x), \Phi^{a b}\left(x^{\prime}\right)\right\}=-2 \delta_{c i}^{a b} \Phi^{c j}\left(x^{\prime}\right) \partial_{j} \delta^{3}\left(x, x^{\prime}\right)+\Phi^{a b}(x) \partial_{i} \delta^{3}\left(x, x^{\prime}\right) \approx 0,
$$

and

$$
\left\{\mathcal{H}_{i}(x), \Phi_{\phi}\left(x^{\prime}\right)\right\}=\Phi_{\phi} \partial_{i} \delta^{3}\left(x, x^{\prime}\right) \approx 0
$$

Combining these results we obtain that

$$
\dot{\Phi}^{i j}=\left\{\Phi^{i j}, H_{Q}\right\} \approx 0
$$


and

$$
\dot{\Phi}_{\phi}=\left\{\Phi_{\phi}, H_{Q}\right\} \approx 0
$$

Furthermore, the Poisson brackets of (42) and (43) among themselves are all zero. Finally, the definitions of the momenta in terms of the velocities remain the same as in the classical case because the quantum potential $Q$ does not depend on the momenta:

$$
\dot{h}_{i j}=\left\{h_{i j}, H_{Q}\right\}=\left\{h_{i j}, H\right\},
$$

and

$$
\dot{\phi}=\left\{\phi, H_{Q}\right\}=\{\phi, H\}
$$

Hence we recover (38) and (39).

We now have a hamiltonian, $H_{Q}$, which generates the bohmian trajectories once the guidance relations (35) and (36) are imposed initially. In the following, we can investigate if the the evolution of the fields driven by $H_{Q}$ forms a four-geometry like in classical geometrodynamics. First we recall a result obtained by Claudio Teitelboim [32]. In this paper, he shows that if the 3-geometries and field configurations defined on hypersurfaces are evolved by some hamiltonian with the form

$$
\bar{H}=\int d^{3} x\left(N \overline{\mathcal{H}}+N^{i} \overline{\mathcal{H}}_{i}\right)
$$

and if this evolution can be viewed as the "motion" of a 3-dimensional cut in a 4-dimensional spacetime (the 3-geometries can be embedded in a fourgeometry), then the constraints $\overline{\mathcal{H}} \approx 0$ and $\overline{\mathcal{H}}_{i} \approx 0$ must satisfy the following algebra 


$$
\begin{aligned}
\left\{\overline{\mathcal{H}}(x), \overline{\mathcal{H}}\left(x^{\prime}\right)\right\} & =-\epsilon\left[\overline{\mathcal{H}}^{i}(x) \partial_{i} \delta^{3}\left(x^{\prime}, x\right)-\overline{\mathcal{H}}^{i}\left(x^{\prime}\right) \partial_{i} \delta^{3}\left(x^{\prime}, x\right)\right] \\
\left\{\overline{\mathcal{H}}_{i}(x), \overline{\mathcal{H}}\left(x^{\prime}\right)\right\} & =\overline{\mathcal{H}}(x) \partial_{i} \delta^{3}\left(x, x^{\prime}\right) \\
\left\{\overline{\mathcal{H}}_{i}(x), \overline{\mathcal{H}}_{j}\left(x^{\prime}\right)\right\} & =\overline{\mathcal{H}}_{i}(x) \partial_{j} \delta^{3}\left(x, x^{\prime}\right)-\overline{\mathcal{H}}_{j}\left(x^{\prime}\right) \partial_{i} \delta^{3}\left(x, x^{\prime}\right)
\end{aligned}
$$

The constant $\epsilon$ in (54) can be \pm 1 depending if the four-geometry in which the 3-geometries are embedded is euclidean $(\epsilon=1)$ or hyperbolic $(\epsilon=-1)$. These are the conditions for the existence of spacetime.

The above algebra is the same as the algebra (24) of GR if we choose $\epsilon=-1$. But the hamiltonian (40) is different from the hamiltonian of GR only by the presence of the quantum potential term $Q$ in $\mathcal{H}_{Q}$. The Poisson bracket $\left\{\mathcal{H}_{i}(x), \mathcal{H}_{j}\left(x^{\prime}\right)\right\}$ satisfies Eq. (56) because the $\mathcal{H}_{i}$ of $H_{Q}$ defined in Eq. (40) is the same as in GR. Also $\left\{\mathcal{H}_{i}(x), \mathcal{H}_{Q}\left(x^{\prime}\right)\right\}$ satisfies Eq. (55) because $\mathcal{H}_{i}$ is the generator of spatial coordinate tranformations, and as $\mathcal{H}_{Q}$ is a scalar density of weight one (remember that $Q$ must be a scalar density of weight one), then it must satisfies this Poisson bracket relation with $\mathcal{H}_{i}$. What remains to be verified is if the Poisson bracket $\left\{\mathcal{H}_{Q}(x), \mathcal{H}_{Q}\left(x^{\prime}\right)\right\}$ closes as in Eq. (54). We now recall the result of Ref. [21]. There it is shown that a general super-hamiltonian $\overline{\mathcal{H}}$ which satisfies Eq. (54), is a scalar density of weight one, whose geometrical degrees of freedom are given only by the three-metric $h_{i j}$ and its canonical momentum, and contains only even powers and no non-local term in the momenta (together with the other requirements, these last two conditions are also satisfied by $\mathcal{H}_{Q}$ because it is quadratic in the momenta and the quantum potential does not contain any non-local term on the momenta), then $\overline{\mathcal{H}}$ must have the following form: 


$$
\overline{\mathcal{H}}=\kappa G_{i j k l} \Pi^{i j} \Pi^{k l}+\frac{1}{2} h^{-1 / 2} \pi_{\phi}^{2}+V_{G}
$$

where

$$
V_{G} \equiv-\epsilon h^{1 / 2}\left[-\kappa^{-1}\left(R^{(3)}-2 \bar{\Lambda}\right)+\frac{1}{2} h^{i j} \partial_{i} \phi \partial_{j} \phi+\bar{U}(\phi)\right] .
$$

With this result we can now establish three possible scenarios for the Bohmde Broglie quantum geometrodynamics, depending on the form of the quantum potential:

\section{1) Quantum geometrodynamics evolution forms a four-geometry}

In this case, the Poisson bracket $\left\{\mathcal{H}_{Q}(x), \mathcal{H}_{Q}\left(x^{\prime}\right)\right\}$ must satisfy Eq. (54). Then $Q$ must be such that $V+Q=V_{G}$ with $V$ given by (29) yielding:

$$
Q=-h^{1 / 2}\left[(\epsilon+1)\left(-\kappa^{-1} R^{(3)}+\frac{1}{2} h^{i j} \partial_{i} \phi \partial_{j} \phi\right)+\frac{2}{\kappa}(\epsilon \bar{\Lambda}+\Lambda)+\epsilon \bar{U}(\phi)+U(\phi)\right] .
$$

Then we have two possibilities:

a) The spacetime is hyperbolic $(\epsilon=-1)$

In this case $Q$ is

$$
Q=-h^{1 / 2}\left[\frac{2}{\kappa}(-\bar{\Lambda}+\Lambda)-\bar{U}(\phi)+U(\phi)\right]
$$

Hence $Q$ is like a classical potential. Its effect is to renormalize the cosmological constant and the classical scalar field potential, nothing more. The quantum geometrodynamics is indistinguishable from the classical one. It is not necessary to require the classical limit $Q=0$ because $V_{G}=V+Q$ already may describe the classical universe we live in. 
b) The spacetime is euclidean $(\epsilon=1)$

In this case $Q$ is

$$
Q=-h^{1 / 2}\left[2\left(-\kappa^{-1} R^{(3)}+\frac{1}{2} h^{i j} \partial_{i} \phi \partial_{j} \phi\right)+\frac{2}{\kappa}(\bar{\Lambda}+\Lambda)+\bar{U}(\phi)+U(\phi)\right] .
$$

Now $Q$ not only renormalize the cosmological constant and the classical scalar

field potential but also change the signature of spacetime. The total potential $V_{G}=V+Q$ may describe some era of the early universe when it had euclidean signature, but not the present era, when it is hyperbolic. The transition between these two phases must happen in a hypersurface where $Q=0$, which is the classical limit.

We can conclude from these considerations that if a quantum spacetime exists with different features from the classical observed one, then it must be euclidean. In other words, the sole relevant quantum effect which maintains the non-degenerate nature of the four-geometry of spacetime is its change of signature to a euclidean one. The other quantum effects are either irrelevant or break completely the spacetime structure. This result points in the direction of Ref. [33].

\section{2) Quantum geometrodynamics evolution is consistent but does} not form a four-geometry

In this case, the Poisson bracket $\left\{\mathcal{H}_{Q}(x), \mathcal{H}_{Q}\left(x^{\prime}\right)\right\}$ does not satisfy Eq. (54) but is weakly zero in some other way. Let us examine some examples.

a) Real solutions of the Wheeler-DeWitt equation.

For real solutions of the Wheeler-DeWitt equation, which is a real equation, the phase $S$ is null. Then, from Eq. (32), we can see that $Q=-V$. 
Hence, the quantum super-hamiltonian (41) will contain only the kinetic term, yielding

$$
\left\{\mathcal{H}_{Q}(x), \mathcal{H}_{Q}\left(x^{\prime}\right)\right\}=0
$$

This is a strong equality. This case is connected with the strong gravity limit of GR [34, 35, 36]. If we take the limit of big gravitational constant $G$ (or small speed of light $c$, where we arrive at the Carroll group [26]), then the potential in the super-hamiltonian constraint of GR can be neglected and we arrive at a super-hamiltonian containing only the kinetic term. The Bohmde Broglie interpretation is telling us that any real solution of the WheelerDeWitt equation yields a quantum geometrodynamics satisfying precisely this strong gravity limit. The classical limit $Q=0$ in this case implies also that $V=0$. It should be interesting to investigate further the structure we obtain here.

b) Non-local quantum potentials.

Any non-local quantum potential breaks spacetime but there are some that may still be consistent. As an example take a quantum potential of the form

$$
Q=\gamma V
$$

where $\gamma$ is a function of the functional $S$ (here comes the non-locality). In the appendix, we exhibit a wave functional solution of a midisuperspace model which yields this type of quantum potential. Let us now calculate $\left\{\mathcal{H}_{Q}(x), \mathcal{H}_{Q}\left(x^{\prime}\right)\right\}:$

$$
\left\{\mathcal{H}_{Q}(x), \mathcal{H}_{Q}\left(x^{\prime}\right)\right\}=\left\{\mathcal{H}(x)+Q(x), \mathcal{H}\left(x^{\prime}\right)+Q\left(x^{\prime}\right)\right\}
$$




$$
=\left\{\mathcal{H}(x), \mathcal{H}\left(x^{\prime}\right)\right\}+\left\{T(x), Q\left(x^{\prime}\right)\right\}+\left\{Q(x), T\left(x^{\prime}\right)\right\}
$$

where $T$ is the kinetic term of the quantum super-hamiltonian. Developing the last two terms we get

$$
\begin{aligned}
\left\{\mathcal{H}_{Q}(x), \mathcal{H}_{Q}\left(x^{\prime}\right)\right\}= & \left\{\mathcal{H}(x), \mathcal{H}\left(x^{\prime}\right)\right\}+\gamma\left\{\mathcal{H}(x), \mathcal{H}\left(x^{\prime}\right)\right\} \\
& -\frac{d \gamma}{d S} V\left(x^{\prime}\right)\left[2 \kappa G_{k l i j}(x) \Pi^{i j}(x) \frac{\delta S}{\delta h_{k l}(x)}+h^{-\frac{1}{2}} \Pi_{\phi}(x) \frac{\delta S}{\delta \phi(x)}\right] \\
& +\frac{d \gamma}{d S} V(x)\left[2 \kappa G_{k l i j}\left(x^{\prime}\right) \Pi^{i j}\left(x^{\prime}\right) \frac{\delta S}{\delta h_{k l}\left(x^{\prime}\right)}+h^{-\frac{1}{2}} \Pi_{\phi}\left(x^{\prime}\right) \frac{\delta S}{\delta \phi\left(x^{\prime}\right)}\right] \\
= & (1+\gamma)\left\{\mathcal{H}(x), \mathcal{H}\left(x^{\prime}\right)\right\} \\
& -\frac{d \gamma}{d S} V\left(x^{\prime}\right)\left[2 \mathcal{H}_{Q}(x)-2 \kappa G_{k l i j}(x) \Pi^{i j}(x)\left(\Pi^{k l}(x)-\frac{\delta S}{\delta h_{k l}(x)}\right)\right. \\
& \left.-h^{-\frac{1}{2}} \Pi_{\phi}(x)\left(\Pi_{\phi}(x)-\frac{\delta S}{\delta \phi(x)}\right)\right] \\
& +\frac{d \gamma}{d S} V(x)\left[2 \mathcal{H}_{Q}\left(x^{\prime}\right)-2 \kappa G_{k l i j}\left(x^{\prime}\right) \Pi^{i j}\left(x^{\prime}\right)\left(\Pi^{k l}\left(x^{\prime}\right)-\frac{\delta S}{\delta h_{k l}\left(x^{\prime}\right)}\right)\right. \\
& \left.-h^{-\frac{1}{2}} \Pi_{\phi}\left(x^{\prime}\right)\left(\Pi_{\phi}\left(x^{\prime}\right)-\frac{\delta S}{\delta \phi\left(x^{\prime}\right)}\right)\right]
\end{aligned}
$$

Using the algebra (24) and the definitions (42) and (43) we have:

$$
\begin{aligned}
\left\{\mathcal{H}_{Q}(x), \mathcal{H}_{Q}\left(x^{\prime}\right)\right\}= & (1+\gamma)\left[\mathcal{H}^{i}(x) \partial_{i} \delta^{3}\left(x, x^{\prime}\right)-\mathcal{H}^{i}\left(x^{\prime}\right) \partial_{i} \delta^{3}\left(x^{\prime}, x\right)\right] \\
& -\frac{d \gamma}{d S} V\left(x^{\prime}\right)\left[2 \mathcal{H}_{Q}(x)-2 \kappa G_{k l i j}(x) \Pi^{i j}(x) \Phi^{k l}(x)-h^{-\frac{1}{2}} \Pi_{\phi}(x) \Phi_{\phi}(x)\right] \\
& +\frac{d \gamma}{d S} V(x)\left[2 \mathcal{H}_{Q}\left(x^{\prime}\right)-2 \kappa G_{k l i j}\left(x^{\prime}\right) \Pi^{i j}\left(x^{\prime}\right) \Phi^{k l}\left(x^{\prime}\right)-h^{-\frac{1}{2}} \Pi_{\phi}\left(x^{\prime}\right) \Phi_{\phi}\left(x^{\prime}\right)\right] \\
& \approx 0
\end{aligned}
$$

The rhs in the last expression is weakly zero because it is a combination of the constraints and the guidance relations (42) and (43). Note that it was 
very important to use the guidance relations to close the algebra. It means that the hamiltonian evolution with the quantum potential (63) is consistent only when restricted to the bohmian trajectories. For other trajectories, it is inconsitent. Concluding, when restricted to the bohmian trajectories, an algebra which does not close in general may close, as shown in the above example. This is an important remark on the Bohm-de Broglie interpretation of canonical quantum cosmology, which sometimes is not noticed.

In the examples above, we have explicitly obtained the "structure constants" of the algebra that caracterizes the "pre-four-geometry" generated by $H_{Q}$ i.e., the foam-like structure pointed long time ago in early works of J. A. Wheeler [30, 37].

\section{3) Quantum geometrodynamical evolution is inconsistent}

In this case, the Poisson bracket $\left\{\mathcal{H}_{Q}(x), \mathcal{H}_{Q}\left(x^{\prime}\right)\right\}$ is not even weakly zero. The quantum geometrodynamics is inconsistent. Note that it may be inconsistent even if the theory is free of anomalies in the sense of section 3. As the Bohm-de Broglie interpretation is a more detailed description of quantum phenomena, its consistency may be more difficult to achieve. The restriction on the quantum potential along these lines may yield natural boundary conditions to the Wheeler-DeWitt equation.

\footnotetext{
${ }^{4}$ One could ask if the guidance relations could not help to close general algebras as in Eq. (54). If this were true, then we could have more general quantum potentials than the ones given in Eq. (59) which would not break spacetime. However, it can be easily checked that this is not possible.
} 


\section{Conclusion and Discussions}

The Bohm-de Broglie interpretation of canonical quantum cosmology yields a quantum geometrodynamical picture where, in general, and always when the quantum potential is non-local, spacetime is broken. The 3-geometries evolved under the influence of a quantum potential do not in general stick together to form a non-degenerate four-geometry. This is not surprising, as it was antecipated long ago [37]. We obtained this result taking a minimally coupled scalar field as the matter source of gravitation, but it can be generalized to any matter source with non-derivative couplings with the metric, like Yang-Mills fields. What is nice with the Bohm-de Broglie approach is that we can investigate further what kind of structure is formed, by means of the Poisson bracket relation (54), and the guidance relations (38) and (39). By assuming the existence of 3-geometries, field configurations, and their momenta, independently on any observations, the Bohm-de Broglie interpretation allows us to use classical tools, like the hamiltonian formalism, to understand the structure of quantum geometry. If this information is useful, we do not know. Already in the two-slit experiment in non-relativistic quantum mechanics, the Bohm-de Broglie interpretation allows us to say from which slit the particle has passed through: if it arrive at the upper half of the screen it must have come from the upper slit, and vice-versa. Such information we do not have in the many worlds interpretation. However, this information is useless: we can neither check it nor use it in other experiments. In canonical quantum cosmology the situation may be the same. The Bohm-de Broglie interpretation yields a lot of information about quantum geometrodynamics 
which we cannot obtain from the many worlds interpretation, but this information may be useless. However, we cannot answer this question precisely if we do not investigate further, and the tools are at our disposal. Furthermore, as the Bohm-de Broglie interpretation is a more detailed description of quantum phenomena, consistency requirements may be more difficult to achieve. As shown in the last section, we can have inconsistent quantum geometrodynamical evolution, even for an anomaly free quantum theory. This is a nice feature of the Bohm-de Broglie interpretation because boundary conditions can then be naturally imposed on solutions of the Wheeler-DeWitt equation. Such boundary conditions would be absent in other interpretations. Also, if we want to be more strict, and impose that quantum geometrodynamics does not break spacetime, then we will have much more stringent boundary conditions. As shown in the previous section, a four-geometry can be attained only if the quantum potential have the the specific form (59). This is a severe restriction on the solutions of the Wheeler-DeWitt equation P. In this case, the sole relevant quantum effect will be a change of signature of spacetime, something pointing towards Hawking's ideas. Our answer to the question in the title is, then, in the affirmative.

In the case of consistent quantum geometrodynamical evolution but with no four-geometry, we have shown that any real solution of the Wheeler-

\footnotetext{
${ }^{5}$ These restrictions on the form of the quantum potential do not occur in minisuperspace models [16] because there the hypersurfaces are restricted to be homogeneous. The only freedom we have is in the time parametrization of the homogeneous hypersurfaces which folliate spacetime. There is a single constraint, which of course always commute with itself irrespective of the quantum potential.
} 
DeWitt equation yields a structure which is the idealization of the strong gravity limit of GR. This type of geometry, which is generate, has already been studied [36]. Due to the generality of this picture (it is valid for any real solution of the Wheeler-DeWitt equation, which is a real equation), it deserves further attention. It may well be that these generate 4-metrics were the correct quantum gemetrodynamical description of the young universe. It would be also interesting to investigate if these structures have a classical limit yielding the usual four-geometry of classical cosmology.

For non-local quantum potentials, we have shown that aparently inconsistent quantum evolutions are in fact consistent if restricted to the bohmian trajectories satisfying the guidance relations (35) and (36). This is a point which is sometimes not taken into account.

Finally, it should be interesting to investigate the connection between the classical limit and the conditions for inflation and/or homogeneity and isotropy of the universe. For instance, neglecting the scalar field, the classical limit of the examples (1-b,2-a,2-b) in the previous section implies that the initial classical hypersurface must have a constant scalar curvature, which is closer to a maximally symmetric initial hypersurface.

We would like to remark that all these results were obtained without assuming any particular factor ordering and regularization of the WheelerDeWitt equation. Also, we did not use any probabilistic interpretation of the solutions of the Wheeler-DeWitt equation. Hence, it is a quite general result. However, we would like to make some comments about the probability issue in quantum cosmology. The Wheeler-DeWitt equation when applied to a closed universe does not yield a probabilistic interpretation for 
their solutions because of its hyperbolic nature. However, it has been suggested many times 17, 38, 39, 40, 41 that at the semiclassical level we can construct a probability measure with the solutions of the Wheeler-DeWitt equation. Hence, for interpretations where probabilities are essential, the problem of finding a Hilbert space for the solutions of the Wheeler-DeWitt equation becomes crucial if someone wants to get some information above the semiclassical level. Of course, probabilities are also useful in the Bohm-de Broglie interpretation. When we integrate the guidance relations (38) and (39), the initial conditions are arbitrary, and it should be nice to have some probability distribution on them. However, as we have seen along this paper, we can extract a lot of information from the full quantum gravity level using the Bohm-de Broglie interpretation, without appealing to any probabilistic notion. In this interpretation, probabilities are not essential. Furthermore, as discussed above, this interpretation may impose severe boundary conditions from which we could extract the important results. Hence, we can take the Wheeler-DeWitt equation as it is, without imposing any probabilistic interpretation at the most fundamental level, but still obtaining information using the Bohm-de Broglie interpretation, and then recover probabilities when we reach the semiclassical level.

It would also be important to investigate the Bohm-de Broglie interpretation for other quantum gravitational systems, like black holes. Attempts in this direction have been made, but within spherical symmetry in empty space [42], where we have only a finite number of degrees of freedom. It should be interesting to investigate more general models. These cases are, however, qualitatively different from quantum closed cosmological models. There is 
no problem in thinking of observers outside an ensemble of black holes. It is quantum mechanics of an open system, with less conceptual problems of interpretation.

The conclusions of this paper are of course limited by many strong assumptions we have tacitly made, as supposing that a continuous threegeometry exists at the quantum level (quantum effects could also destroy it), or the validity of quantization of standard GR, forgetting other developments like string theory. However, even if this approach is not the apropriate one, it is nice to see how far we can go with the Bohm-de Broglie interpretation, even in such incomplete stage of canonical quantum gravity. It seems that the Bohm-de Broglie interpretation may at least be regarded as a nice "gauge" 43, to be used in quantum cosmology, as, probably, it will prove harder, or even impossible, to reach the detailed conclusions of this paper using other interpretations. However, if the finer view of the Bohm-de Broglie interpretation of quantum cosmology can yield useful information in the form of observational effects, then we will have means to decide between interpretations, something that will be very important not only for quantum cosmology, but for quantum theory itself.

\section{ACKNOWLEDGEMENTS}

We would like to thank Brandon Carter and the Cosmology Group of CBPF for useful discussions. Our special warm thanks are to Karel Kuchař for many helpful and lucid comments on our work while he was visiting our institute. We would also like to thank CNPq of Brazil for financial support. 


\section{Appendix: A Non-local Quantum Potential in the Bohm-de Broglie Interpretation of a Quantum Spherical Spacetime}

We present here an example where a quantum potential of the type (2-b) is obtained. It is an spherically symmetric midisuperspace model with an electromagnetic field. A factor-ordering is proposed just to yield an exact solution of the Wheeler-DeWitt equation, which is not regularized].

We start from the ADM decomposition of the general spherically symmetric electrovacuum spacetime metric on the manifold $R \times R \times S^{2}$ :

$$
d s^{2}=-N^{2} d t^{2}+\Lambda^{2}\left(d r+N^{r} d t\right)^{2}+R^{2} d \Omega^{2}
$$

where $N, N^{r}$ are the lapse and shift functions respectively (both dependent on $r$ and $t$ ), and $d \Omega$ denotes the standard line element on $S^{2}$. The electromagnetic potential is taken to be described by the spherically symmetric one-form:

$$
d A=\Gamma(r, t) d r+\Phi(r, t) d t
$$

The ADM action in midisuperspace, after integrating over the two sphere, reads $(c \equiv 1)$

\footnotetext{
${ }^{6}$ In fact, the model studied here can be reduced to a minisuperspace model, with a finite number of degrees of freedom [44]. However, our goal with this appendix is just to argue that quantum potentials of the type (2-b) are not so difficult to appear in canonical quantum gravity. They may be obtained already from spherically symmetric WheelerDeWitt equations, as we will see.
} 


$$
\begin{array}{r}
S=\int d t \int d r \frac{1}{2 N}\left\{\frac { 1 } { G } \left[N^{2} \Lambda-\Lambda \dot{R}^{2}+2 \frac{N^{2} R \Lambda^{\prime} R^{\prime}}{\Lambda^{2}}-2 \frac{N^{2} R R^{\prime \prime}}{\Lambda}-\right.\right. \\
\Lambda\left(N^{r}\right)^{2} R^{\prime 2}+2 N^{r} \dot{R}(\Lambda R)^{\prime}-2 R N^{r} R^{\prime}\left(\Lambda N^{r}\right)^{\prime}+2 R \Lambda\left(N^{r}\right)^{\prime} \dot{R} \\
\left.\left.+2 R N^{r} \dot{\Lambda} R^{\prime}-2 R \dot{\Lambda} \dot{R}-N^{2} \frac{R^{\prime 2}}{\Lambda}\right]+\frac{R^{2}}{\Lambda}\left(\dot{\Gamma}-\Phi^{\prime}\right)^{2}\right\},
\end{array}
$$

where a prime denotes a derivative in $r$. Varying the action with respect to $N$ and $N^{r}$ leads to the super-hamiltonian and the super-momentum constraints [44, 45]

$$
\mathcal{H} \equiv \frac{G}{2} \frac{\Lambda P_{\Lambda}^{2}}{R^{2}}-G \frac{P_{\Lambda} P_{R}}{R}+\frac{V_{g}}{G}+\frac{\Lambda P_{\Gamma}^{2}}{2 R^{2}} \approx 0,
$$

and

$$
\mathcal{H}_{r} \equiv P_{R} R^{\prime}-\Lambda P_{\Lambda}^{\prime} \approx 0,
$$

where

$$
\frac{V_{g}}{G}=\frac{R R^{\prime \prime}}{\Lambda}-\frac{R R^{\prime} \Lambda^{\prime}}{\Lambda^{2}}+\frac{R^{\prime 2}}{2 \Lambda}-\frac{\Lambda}{2}
$$

Varying the action with respect to the Lagrange multiplier $\phi$ leads to

$$
P_{\Gamma}^{\prime} \approx 0 .
$$

Boundary conditions for all fields are assumed to hold such that all integrals are well defined, and such that the classical spacetime metric is nondegenerate [45].

We perform the quantization in the standard formal manner. All constraints act on wave functionals $\Psi[\Lambda(r), R(r), \Gamma(r)]$. The electromagnetic 
constraint (72) is solved [46] by $\Psi=f\left(\int_{\infty}^{\infty} \Gamma d r\right) \psi[\Lambda(r), R(r)]$, where $f$ is an arbitrary differentiable function.

From the hamiltonian constraint, we obtain the Wheeler-DeWitt equation with a particular factor ordering:

$$
\left(-\frac{G \hbar^{2} \Lambda}{2 R^{2}} F \frac{\delta}{\delta \Lambda} F^{-1} \frac{\delta}{\delta \Lambda}+\frac{G \hbar^{2}}{R} F \frac{\delta}{\delta R} F^{-1} \frac{\delta}{\delta \Lambda}+\frac{V_{g}}{G}-\frac{\hbar^{2} \Lambda \delta^{2}}{2 R^{2} \delta \Gamma^{2}}\right) \Psi=0
$$

where

$$
F \equiv R \sqrt{\left(\frac{R^{\prime}}{\Lambda}\right)^{2}+\frac{2 m}{R}-\frac{q^{2}}{R^{2}}-1}
$$

Our solution has the form 46]

$$
\Psi=e^{\frac{i q}{\hbar} \int_{-\infty}^{\infty} \Gamma d r} \psi[\Lambda(r), R(r)]
$$

where the gravitational and electromagnetic degrees of freedom are separated, and $\psi$ satisfies a reduced Wheeler-DeWitt equation

$$
\left(-\frac{G \hbar^{2} \Lambda}{2 R^{2}} F \frac{\delta}{\delta \Lambda} F^{-1} \frac{\delta}{\delta \Lambda}+\frac{G \hbar^{2}}{R} F \frac{\delta}{\delta R} F^{-1} \frac{\delta}{\delta \Lambda}+\frac{V_{g}}{G}+\frac{\Lambda q^{2}}{2 R^{2}}\right) \psi=0 .
$$

We are using this particular factor ordering because in this case there is an exact solution known in the literature [46], which reads

$$
\psi=\exp \frac{i S_{0}}{\hbar}
$$

where 


$$
S_{0}=G^{-1} \int_{-\infty}^{\infty} d r\left\{\Lambda F-\frac{1}{2} R R^{\prime} \ln \frac{\frac{R^{\prime}}{\Lambda}+\frac{F}{R}}{\frac{R^{\prime}}{\Lambda}-\frac{F}{R}}\right\}
$$

It is easy to see that, as the Wheeler-DeWitt equation is real, the complex conjugate of the wave functional (77) is another exact solution of it. Hence, we have two independent exact solutions of the Wheeler-DeWitt equation, and because of its linearity, any linear superposition of them will also be a solution :

$$
\psi=a \exp \left(\frac{i S_{0}}{\hbar}\right)+b \exp \left(\frac{-i S_{0}}{\hbar}\right)
$$

Writing the wave functional in polar form

$$
\psi=A \exp \frac{i S}{\hbar}
$$

and replacing it in the Wheeler-DeWitt equation (76), we obtain two equations. One of them reads

$$
\frac{G \Lambda}{2 R^{2}}\left(\frac{\delta S}{\delta \Lambda}\right)^{2}-\frac{G}{R} \frac{\delta S}{\delta \Lambda} \frac{\delta S}{\delta R}+V+Q=0
$$

where $V$ stands for the classical potential

$$
V \equiv \frac{V_{g}}{G}+\frac{\Lambda q^{2}}{2 R^{2}}
$$

and $Q$ is the quantum potential

$$
Q=\frac{G \hbar^{2}}{A R}\left(-\frac{\Lambda \delta^{2} A}{2 R \delta \Lambda^{2}}+\frac{\delta^{2} A}{\delta R \delta \Lambda}+\left(-\frac{1}{F} \frac{\delta F}{\delta R}+\frac{\Lambda}{2 R F} \frac{\delta F}{\delta \Lambda}\right) \frac{\delta A}{\delta \Lambda}\right)
$$

For the wave functional $(79)$, the quantum potential (83) reads 


$$
Q=\gamma V
$$

where $V$ is the classical potential, and the factor $\gamma$ is given by

$$
\gamma=-4\left\{\left(\frac{a b}{A^{2}}\right)^{2} \sin ^{2}\left(\frac{2 S_{0}}{\hbar}\right)+\frac{a b}{A^{2}} \cos \left(\frac{2 S_{0}}{\hbar}\right)\right\} .
$$

As the phase functional $S$ is given in terms of $S_{0}$ by $S=\frac{\hbar}{2 i} \ln \left(\frac{\psi}{\psi^{*}}\right)$, where $\psi$ is a function of $S_{0}$ only, then

$$
\gamma=\gamma(S)
$$

Hence we have found a concrete example of a quantum potential of the type studied in (2-b), where the constraint's algebra close with new "structure constants", breaking the four-geometry of spacetime.

\section{References}

[1] N. Bohr, Atomic Physics and Human Knowledge (Science Editions, New York, 1961); N. Bohr; Phys. Rev. 48, 696 (1935).

[2] W. Heisenberg, The Physical Principles of the Quantum Theory (Dover, New York, 1949).

[3] J. von Neumann, Mathematical Foundations of Quantum Mechanics (Princeton University Press, Princeton, 1955).

[4] R. Omnès, The Interpretation of Quantum Mechanics (Princeton University Press, Princeton, 1994). 
[5] V.F Mukhanov, in Physical Origins of Time Asymmetry, ed by J. J. Halliwell, J. Pérez-Mercader and W. H. Zurek (Cambridge University Press, 1994).

[6] H. D. Zeh, in Decoherence and the Appearance of a Classical World in Quantum Theory (Springer-Verlag, Berlin, 1996).

[7] M. Gell-Mann and J. B. Hartle, in Complexity, Entropy and the Physics of Information, ed. by W. Zurek (Addison Wesley, 1990).

[8] G.C. Ghirardi, A. Rimini and T. Weber, Phys. Rev. D 34470 (1986); G.C. Ghirardi, P. Pearle and A. Rimini, Phys. Rev.A 42, 78 (1990).

[9] R. Penrose, in Quantum Implications: Essays in Honour of David Bohm, ed. by B. J. Hiley and F. David Peat (Routledge, London, 1987).

[10] The Many-Worlds Interpretation of Quantum Mechanics, ed. by B. S. DeWitt and N. Graham (Princeton University Press, Princeton, 1973).

[11] D. Bohm, Phys. Rev. 85, 166 (1952); D. Bohm, B. J. Hiley and P. N. Kaloyerou, Phys. Rep. 144, 349 (1987).

[12] P. R. Holland, The Quantum Theory of Motion: An Account of the de Broglie-Bohm Causal Interpretation of Quantum Mechanichs (Cambridge University Press, Cambridge, 1993).

[13] J. C. Vink, Nucl. Phys. B369, 707 (1992).

[14] Y. V. Shtanov, Phys. Rev. D54, 2564 (1996). 
[15] A. Valentini, Phys. Lett. A158, 1, (1991).

[16] J. A. de Barros and N. Pinto-Neto, Int. J. of Mod. Phys. D7, 201 (1998).

[17] J. Kowalski-Glikman and J. C. Vink, Class. Quantum Grav. 7, 901 (1990).

[18] E. J. Squires, Phys. Lett. A162, 35 (1992).

[19] J. A. de Barros, N. Pinto-Neto and M. A. Sagioro-Leal, Phys. Lett. A241, 229 (1998).

[20] R. Colistete Jr., J. C. Fabris and N. Pinto-Neto, Phys. Rev. D57, 4707 (1998).

[21] S. A. Hojman, K. Kuchař and C. Teitelboim, Ann. Phys. 96, 88 (1976).

[22] N. C. Tsamis and R. P. Woodward, Phys. Rev. D36, 3641 (1987).

[23] K. Maeda and M. Sakamoto, Phys. Rev. D54, 1500 (1996).

[24] T. Horiguchi, K. Maeda and M. Sakamoto, Phys. Lett. B344, 105 (1995).

[25] J. Kowalski-Glikman and K. A. Meissner, Phys. Lett. B376, 48 (1996).

[26] J. M. Lévy Leblond, Ann. Inst. Henri Poincarè 3, 1 (1965).

[27] D. Bohm and J. P. Vigier, Phys. Rev. 96, 208 (1954).

[28] A. Valentini, Phys. Lett. A156, 5 (1991).

[29] P. N. Kaloyerou, Phys. Rep. 244, 287 (1994). 
[30] J.A. Wheeler, in Battelle Rencontres: 1967 Lectures in Mathematical Physics, ed. by B. DeWitt and J.A.Wheeler (Benjamin New York, 1968).

[31] B. S. DeWitt, Phys. Rev. 160, 1113 (1967).

[32] C. Teitelboim, Ann. Phys. 80, 542 (1973).

[33] Euclidean Quantum Gravity, ed. by G. W. Gibbons and S. W. Hawking (World Scientific, London, 1993).

[34] C. Teitelboim, Phys. Rev. D 253159 (1982).

[35] M. Henneaux, M. Pilati and C. Teitelboim, Phys. Lett.110 B 123 (1982)

[36] G. Dautcourt, report gr-gc/9801093.

[37] J. A. Wheeler, Ann. Phys. 2, 604 (1957); J. A. Wheeler, in Relativity, Groups and Topology, ed. by B. DeWitt and C. DeWitt (Gordon and Breach, New York, 1964); G.M Patton and J.A Wheeler, in Quantum Gravity. An Oxford Symposium, ed. by C.J. Isham, R.Penrose and D. Sciama (Clarendon Press, Oxford, 1975).

[38] T. Banks, Nucl. Phys. B249, 332 (1985).

[39] T. P. Singh and T. Padmanabhan, Ann. Phys. 196, 296 (1989).

[40] D. Giulini and C. Kiefer, Class. Quantum Grav. 12, 403 (1995).

[41] J.J. Halliwell, in Quantum Cosmology and Baby Universes, ed. by S. Coleman, J.B. Hartle, T. Piran and S. Weinberg (World Scientific, Singapore, 1991). 
[42] M. Kenmoku, H. Kubotani, E. Takasugi and Y. Yamazaki, report gr-qd 9810039 .

[43] We thank this image to Brandon Carter.

[44] K. Kuchař, Phys. Rev. D 50, 3961 (1994).

[45] J. Louko and S. N. Winters-Hilt, Phys. Rev.D 54, 2647 (1996).

[46] T. Brotz and C. Kiefer, Phys. Rev. D 55, 2186 (1997). 